\title{
A REDE DE ATENÇÃO ESPECIALIZADA EM FISIOTERAPIA NEUROPEDIÁTRICA DO ESTADO DO RIO DE JANEIRO: ASPECTOS DA PRÁTICA CLÍNICA E CAPACITAÇÃO PROFISSIONAL
}

\author{
THE NETWORK OF SPECIALIZED CARE IN NEUROPEDIATRIC PHYSIOTHERAPY \\ IN THE STATE OF RIO DE JANEIRO: ASPECTS OF CLINICAL PRACTICE AND PROFESSIONAL TRAINING
}

\section{Miriam Ribeiro Calheiros de Sá Carla Trevisan Martins Ribeiro Adelino Furtado Madureira Maria Fernanda Negreiros Vieira Nicolette Celani Cavalcanti Paula de Almeida Thomazinho Fabiano Luiz dos Santos}

Instituto Nacional de Saúde da Mulher, da Criança e do Adolescente Fernandes Figueira

\section{RESUMO}

Nos últimos anos, nota-se uma clara mudança no perfil epidemiológico das doenças infantojuvenis no Brasil, com um efetivo aumento da população acometida por condições crônicas e complexas de saúde. 0 que se nota é que a rede de atenção fisioterapêutica pediátrica ainda é incipiente, em face das demandas dessa população, não sendo encontrados estudos sob este prisma. Dessa forma, o objetivo desta pesquisa foi conhecer a rede de assistência fisioterapêutica neuropediátrica no estado do Rio de Janeiro, assim como outras questões da prática clínica, como o tipo de tratamento oferecido e a capacitação do profissio-

\author{
Contato \\ Miriam Ribeiro Calheiros de Sá \\ Instituto Nacional de Saúde da Mulher, da Criança e \\ do Adolescente Fernandes Figueira. \\ E-mail: calheirosa@uol.com.br
}

\begin{abstract}
In recent years, there has been a clear change in the epidemiological profile of pediatric diseases in Brazil, with an effective increase in the population affected by chronic and complex health conditions. It is observed that the network of pediatric physical therapy is still incipient, given the demands of such population, and no studies were found under this prism. Thus, the objective of this research is understand the neuropediatric physiotherapeutic care network in the State of Rio de Janeiro, as well as other issues of clinical practice, such as the type of treatment offered and the qualification of the professio-
\end{abstract}

nal envolvido na reabilitação infantil. Este foi um estudo analítico e transversal, realizado entre os anos de 2013 e 2016, nas unidades de saúde que atendem ao Sistema Único de Saúde (SUS) nos municípios do estado do Rio de Janeiro e que ofereciam tratamento de fisioterapia para crianças e adolescentes. Os dados da presente pesquisa apontam para uma heterogeneidade na prestação do serviço fisioterapêutico pediátrico e na capacitação dos seus profissionais, reforçando a necessidade de um programa de capacitação/educação continuada para os fisioterapeutas pediátricos atuarem nas unidades do SUS.

Palavras-chave: Criança. Adolescente. Fisioterapia. Doença Crônica. nal involved in child rehabilitation. This was an analytical and cross-sectional study carried out between the years of 2013 and 2016, in the health units that attend the Brazilian Unified Health System (SUS) in the municipalities of the State of Rio de Janeiro, and that offered physical therapy treatment for children and adolescents. The data of the present research point to a heterogeneity in the provision of the pediatric physiotherapeutic service and in the training of its professionals, reinforcing the need for a training / continuing education program for pediatric physiotherapists to work in the units of the SUS.

Keywords: Child. Adolescent. Physical Therapy Specialty. Chronic Disease. 


\section{INTRODUÇÃO}

$\mathrm{N}$ os últimos 15 anos, nota-se uma mudança no perfil epidemiológico das doenças infantojuvenis brasileiras, com uma rápida transição das condições de saúde, em que ainda convivem condições agudas e condições crônicas de saúde dessa população ${ }^{1}$. As doenças crônicas não transmissíveis constituem hoje o maior desafio para os sistemas de saúde por modificarem o padrão de necessidades da população, o que lhe impõe uma configuração sensível à produção da equidade e com capacidade de resolutividade dos problemas, não mais na perspectiva de cura, mas do cuidado. Dados da Organização Mundial da Saúde (OMS) revelam que as doenças crônicas não transmissíveis responderam, em 2011, por $59 \%$ da mortalidade total do mundo, havendo uma estimativa de ultrapassar os $78 \%$ já no ano de $2020^{2}$.

O termo crianças com necessidades de saúde especiais (CRIANES) foi definido, em 1988, para designar aquelas crianças que têm ou estão em maior risco de apresentarem uma alteração crônica, seja no âmbito físico, seja do desenvolvimento, e que requerem cuidados de saúde de determinado tipo ou em quantidade superior ao que é exigido por crianças em geral ${ }^{3}$. No entanto, apesar de $13 \%$ a $18 \%$ de crianças serem consideradas como tendo necessidades, há uma variação considerável na complexidade clínica nas limitações funcionais e necessidade de recursos entre as CRIANES ${ }^{4}$.

Nota-se, portanto, uma inconsistência na maneira como essas crianças e adolescentes são definidos pela literatura. No presente artigo, tomar-se-á como base o conceito de condições crônicas de saúde em crianças e adolescentes de Cohen e colaboradoes4 (2011), proposto a partir de uma recomendação baseada em revisão sistemática sobre o assunto. Nesse trabalho, os autores propõem quatro amplos domínios para caracterizar doenças crônicas da infância: necessidades; condições crônicas; limitações funcionais; e utilização de cuidados em saúde. Eles chamam a atenção para uma revisão realizada por Feudtner e colaboradores (2000), que compilou uma lista da Classificação Internacional de Doenças, na qual o código "condições crônicas complexas" (CCC) foi estabelecido com base em uma definição operacional de uma condição clínica que tem a duração de mais do que 12 meses e envolve diferentes sistemas ou órgãos, exigindo um elevado nível de cuidados especiais e internação ${ }^{4}$.

As crianças com CCC necessitam, portanto, de assistência integral de saúde desde os primeiros dias de vida, com especial atenção a ações de estimulação do desenvolvimento sensório-motor preco- $c^{5}$. A estimulação ou intervenção precoce abrange uma variedade de estímulos para auxiliar o desenvolvimento motor e cognitivo de lactentes e crianças na faixa etária de zero a 3 anos, envolvendo tipicamente terapias tradicionais como fisioterapia, terapia ocupacional e fonoaudiologia ${ }^{6-7}$. Embora esse tipo de estimulação não seja puramente uma técnica fisioterapêutica, a fisioterapia parece ser primordial para tratamento de crianças que apresentem sequelas predominantemente motoras $^{8}$.

A partir da premissa do Sistema Único de Saúde (SUS) brasileiro em que a saúde é um direito de todos e dever do Estado, entende-se que programas de assistência à saúde infantil devem ser ofertados pelo sistema de saúde do País ${ }^{9}$.

O SUS encontra-se organizado a partir de um modelo de atenção à saúde que pressupõe a integração dos serviços interfederativos - por meio das redes de atenção, regionalização (região de saúde) e hierarquização (níveis de complexidade dos serviços). Estes são os pilares que sustentam o modelo de atenção à saúde, conforme dispõe o art. 198 da Constituição Federal. A Carta Magna, ao estabelecer que o SUS é um sistema integrado, organizado em rede regionalizada e hierarquizada, definiu o modelo de atenção à saúde e a sua forma organizativa ${ }^{10}$.

O SUS, da forma como está estruturado, mostra-se mais preparado para o cuidado das condições agudas presentes do que para o cuidado clínico das condições crônicas. A assistência à saúde de pessoas com CCC constitui um processo complexo que envolve o desenvolvimento de práticas de autocuidado, abordagens multiprofissionais e garantia de continuidade assistencial, o que só pode ser obtido por meio de sistemas integrados ${ }^{11}$.

Surgiram, assim, propostas de estruturação de redes de atenção à saúde, visando à consolidação de sistemas de saúde integrados que favorecem o acesso com continuidade assistencial, a integralidade da atenção e a utilização racional dos recursos existentes ${ }^{12}$. Em Portaria editada pelo Ministério da Saúde, consta a seguinte definição de Rede de Atenção à Saúde: "arranjos organizativos de ações e serviços de saúde, de diferentes densidades tecnológicas, que, integradas por meio de sistemas de apoio técnico, logístico e de gestão, buscam garantir a integralidade do cuidado"11.

Pensando no cuidado multidisciplinar de crianças com CCC, que muitas vezes perpassa por ações de reabilitação, observa-se que houve pouco progresso para a garantia da assistência integral a essa população. Para tentar melhorar a assistência, foram criados, pelo Governo Federal, diversos programas e ações de saúde em que se enfatizava a importân- 
cia da intervenção precoce multiprofissional para a reabilitação da criança em toda a sua capacidade funcional9,13. Contudo, apesar da criação de alguns centros especializados, os serviços de reabilitação brasileiros têm cobertura assistencial baixa e organizada por tipo de deficiência, excluindo a assistência aos casos de deficiência múltipla ${ }^{14-16}$.

A partir do Programa de Atenção à Saúde da Pessoa Portadora de Deficiência, criado pelo Ministério da Saúde, houve um incentivo para a criação de centros de reabilitação especializados. Esse documento apresenta a intervenção precoce e, nesta, insere o tratamento fisioterapêutico como um instrumento de prevenção destinado a impedir a instalação de deficiências motoras, mentais ou sensoriais ${ }^{14}$.

Posteriormente, o Ministério da Educação e Cultura lançou um documento intitulado "Diretrizes Educacionais para Estimulação Precoce", em que ressaltou a importância dos programas de estimulação precoce para crianças com necessidades especiais e elaborou diretrizes e normas que fundamentavam esses programas, com relação ao tipo, tempo e frequência de atendimentos das terapias ${ }^{17}$.

A Política Nacional para Integração da Pessoa Portadora de Deficiência somente foi regulamentada em 1999, por intermédio do Decreto $n^{\circ} 3.29818$. Essa política estabelecia em seu art. 16, inciso III que:

[...] cabe aos órgãos federais direta ou indiretamente, a criação de rede de serviços regionalizados, descentralizados e hierarquizados em crescentes níveis de complexidade, voltada ao atendimento à saúde e reabilitação da pessoa portadora de deficiência, articulada com os serviços sociais, educacionais e com o trabalho.

A partir disso, iniciou-se a construção de programas de assistência para reabilitação e, posteriormente, o início de uma organização em rede de atenção, em que o tratamento fisioterapêutico destinado a crianças estava inserido como um dos pilares básicos ${ }^{13}$.

Já em 2010, o Ministério da Saúde brasileiro estabeleceu as diretrizes para a organização da Rede de Atenção à Saúde no âmbito do SUS ${ }^{19}$. Finalmente, em 2012, o Ministério da Saúde, por meio da Portaria $n^{\circ} 793^{20}$, instituiu a Rede de Cuidados à Pessoa com Deficiência no âmbito do SUS, constituída pela atenção básica, atenção especializada em reabilitação e atenção hospitalar de urgência e emergência.

Como pode ser observado, o governo federal brasileiro estabeleceu critérios e diretrizes ge- rais para os serviços de reabilitação, com relação a modelos de atendimento e organização da rede de serviços, porém a adequação e implementação dessas normatizações ficou a cargo dos gestores estaduais e municipais de acordo com a necessidade e realidade locais. No entanto, apesar dos avanços na construção dessa rede de serviços, ainda persistem questões que dificultam a obtenção de um cuidado verdadeiramente integral, em especial para crianças com CCC ${ }^{5,13}$.

O que se observa, relativo ao cuidado de crianças e adolescentes com CCC, é a presença de um maior risco de que ocorra o cuidado em saúde de maneira fragmentada, com falta de coordenação das ações, podendo se caracterizar por atendimento em diferentes instituições com duplicação de exames e planejamento desse cuidado sem que haja comunicação entre as partes envolvidas ${ }^{5 ; 16}$.

Desse modo, as informações epidemiológicas podem ser valiosas, atenuando as desigualdades de cobertura assistencial à saúde e adequando a assistência. Todavia, não existem estudos sob este prisma que evidenciem a rede de assistência fisioterapêutica neurofuncional para crianças, principalmente no caso das CCC, assim como outras questões da prática clínica, como o tipo de tratamento oferecido e a capacitação do profissional envolvido na reabilitação infantil.

Partindo dessas considerações, esta pesquisa objetivou mapear e conhecer a rede de atenção fisioterapêutica neuropediátrica no estado do Rio de Janeiro, assim como verificar o tipo de atendimento oferecido e a qualificação do profissional sobre a atuação fisioterapêutica na área.

Acredita-se que o conhecimento referente a essa rede de atenção pode servir de base para a construção ou aprimoramento dessa assistência, a fim de contemplar todas as necessidades da clientela infantil.

\section{MÉTODO}

$\mathrm{E}$ ste foi um estudo analítico e transversal, nas unidades de saúde que atendem ao SUS nos municípios do estado do Rio de Janeiro, e que ofereciam tratamento de fisioterapia para crianças. Inicialmente, foram selecionados os municípios participantes a partir do quantitativo populacional (população acima de 200 mil habitantes) ou pela sua representatividade regional no estado, conforme Censo IBGE 2010.

Dessa forma, foram selecionados dois municípios de cada Região do estado (Norte Fluminense, Noroeste Fluminense, das Baixadas Litorâneas, 
Serrana, Médio Paraíba, Centro-Sul Fluminense), exceto na Região Metropolitana, onde foram visitados todos os municípios que ofereciam esse tipo de atendimento.

A partir da seleção dos municípios, foi feito um contato com as Secretarias Municipais de Saúde (SMS) e realizada busca ativa nos sites das SMS e no Cadastro Nacional de Estabelecimentos de Saúde (CNES), a fim de obter uma listagem das unidades de saúde públicas que ofereciam atendimento ambulatorial de fisioterapia à população infantil. Quando o município selecionado não possuía ao menos três unidades públicas, foram visitadas todas as unidades particulares e filantrópicas que recebiam subsídio/repasse do SUS para tal fim. Após a confecção da relação de serviços, foi realizado um primeiro contato telefônico para confirmar se esses locais, de fato, realizavam atendimento fisioterapêutico neurofuncional para crianças.

Em relação aos serviços do município do Rio de Janeiro, optou-se pela inclusão de visita somente às unidades pertencentes aos Núcleos de Atenção Interdisciplinar do Desenvolvimento Infantil (NAIDI), criados para executar as ações de reabilitação no município e constituída atualmente por sete núcleos. Os NAIDI estão distribuídos nas áreas de planejamento (AP) (ou programáticas) do município do Rio de Janeiro e contam com equipes multidisciplinares para o atendimento ambulatorial de crianças com desvios do desenvolvimento sensório-motor ${ }^{13,21-22}$.

A partir da informação advinda dessas fontes, foram marcadas visitas técnicas e entrevistas com os chefes de serviços e com os fisioterapeutas dessas instituições a fim de conhecer o atendimento realizado, bem como a capacitação profissional dos fisioterapeutas, com o preenchimento de dois questionários elaborados com perguntas estruturadas e semiestruturadas específicas a cada entrevistado para coleta de dados sobre os objetos de pesquisa. Com relação à prática clínica, foram pesquisadas as seguintes variáveis: tipo de instituição, tipo de vínculo com o SUS, metodologia de trabalho fisioterapêutico, forma de atendimento, frequência e duração das sessões de tratamento. Já em relação aos profissionais, as varáveis foram: tempo de formado, especialização para trabalhar com crianças, participação em eventos ou atualizações em fisioterapia neurofuncional pediátrica, evolução das práticas pediátricas e dificuldades para realização de atualizações.

Os dados foram coletados entre julho de 2013 e agosto de 2016, armazenados no programa EPIINFO 2000 e analisados de forma descritiva, separando a amostra em dois grupos (Região Metropolitana e demais Regiões do estado), comparando os achados encontrados.

O projeto foi submetido e aprovado pelo Comitê de Ética em Pesquisa do Instituto Fernandes Figueira - IFF/ Fiocruz sob o número CAAE 0083.0.008.000-11. 


\section{RESULTADOS}

$\mathrm{D}$ e acordo com os critérios de inclusão, foram selecionados previamente 21 municípios do estado do Rio de Janeiro. Contudo, após o mapeamento das instituições de cada município, três foram excluídos da pesquisa - a saber: São João de Meriti, Belford Roxo e Magé, pois não foram localizados centros de tratamento fisioterapêutico ambulatorial pediátrico que pertencessem ou recebessem repasse do SUS. Desse modo, foram visitados 17 municípios, sendo 5 na região metropolitana e 12 nas demais regiões do estado.

A partir da seleção dos municípios, 44 instituições compuseram a amostra: 18 na região metropolitana e 26 nas demais regiões. O número de instituições visitadas em cada município variou de um a três, exceto o município do Rio de Janeiro, onde foram visitadas sete instituições.

Quando analisados os grupos Região Metropolitana e demais Regiões do estado, observou-se maior concentração de instituições no primeiro grupo.

\section{Instituições: modelos e diretrizes utilizadas no trata- mento fisioterapêutico pediátrico}

A tabela 1, que apresenta a distribuição dos grupos com os respectivos municípios, bem como das instituições, tipo de instituição visitada com relação ao vínculo com SUS (instituições próprias ou conveniadas), mostra claramente a carência de instituições na Região Noroeste Fluminense e um maior percentual de instituições próprias do SUS na Região Metropolitana.

Tabela 1. Distribuição das instituições pesquisadas por grupos, municípios e tipo de vínculo com SUS. Rio de Janeiro, 2018

Grupos

Metropolitana

Demais Regiões

Total
Região / Municípios

Rio de Janeiro, Niterói, São Gonçalo, Itaboraí, Duque de Caxias e Nova Iguaçu

Norte Fluminense (Campos dos Goytacazes e Macaé) Noroeste Fluminense (Itaperuna e São José de Ubá)

Serrana (Petrópolis e Teresópolis)

Baixadas Litorâneas (Cabo frio e Maricá)

Médio Paraíba (Volta Redonda e Angra dos Reis)

Centro-Sul Fluminense (Vassouras e Três Rios) $\begin{array}{ll}\text { Repasse SUS } & \text { SUS } \\ \mathbf{f}(\%) & \mathbf{f}(\%)\end{array}$

$6(33,3 \%) \quad 12(66,7 \%)$

6

$1-1$

$4-1$

$1 \quad 3$

14

$3-1$

$10(38,5 \%) \quad 16(61,5 \%)$

Nota: $f=$ frequência; $\%=$ percentual 
Quanto à assistência fisioterapêutica oferecida nas diversas instituições, observou-se que em ambos os grupos a metodologia de trabalho fisioterapêutico mais prevalente foi o conceito neuroevolutivo, associado ou não a outras técnicas ou métodos de tratamento. Contudo, seis instituições não souberam informar a metodologia de tratamento empregada, sendo uma da Região Metropolitana e cinco nas demais Regiões (tabela 2).

Ainda em relação às diretrizes de atendimento (tabela 2), somente uma instituição da Região Metro- politana realizava sessão de tratamento exclusivamente em grupo, todas as outras ofereciam atendimento individual, sendo que algumas com ambas as possibilidades. As sessões de terapia recomendadas eram, na maior parte da amostra, de duas vezes por semana, havendo diferença mais relevante entre os grupos somente com relação ao tempo de duração de cada sessão. Toda a amostra referiu que orientava frequentemente os responsáveis pelas crianças quanto ao tratamento e exercícios domiciliares.

Tabela 2. Distribuição das instituições com relação à metodologia de trabalho, forma de atendimento, frequência e duração das sessões de tratamento. Rio de Janeiro, 2018

Variável

Metodologia de trabalho

Conceito Neuroevolutivo

Outros

Não sabe informar

Tipo de atendimento

Individual

Individual + grupo

Grupo

Frequência de atendimento

1 vez por semana

2 vezes por semana

3 vezes por semana

Duração das sessões
Região metropolitana

$$
\begin{aligned}
& 15(83 \%) \\
& 2(11 \%) \\
& 1(6 \%)
\end{aligned}
$$

17 (94\%)

$7(39 \%)$

$1(6 \%)$

$5(6 \%)$
$13(94 \%)$
-

$12(67 \%)$

$4(22 \%)$

$2(11 \%)$

\section{Demais regiões}

$19(73 \%)$

$2(8 \%)$

$5(19 \%)$

$26(100 \%)$

7 (27\%)

$1(4 \%)$

$24(92 \%)$

$1(4 \%)$

$8(31 \%)$

$8(31 \%)$

$8(31 \%)$

$2(7 \%)$

\section{Perfil dos profissionais fisioterapeutas}

Com relação aos fisioterapeutas, foi entrevistado um total de 75 profissionais, sendo 29 da Região Metropolitana e 46 das demais Regiões do estado. O tempo de formado variou de 2 a 35 anos, com mediana de tempo de 15,36 anos no grupo da Região Metropolitana e de 13,73 anos no outro grupo.

A maioria dos fisioterapeutas de ambos os grupos tinha algum tipo de formação específica para trabalhar com crianças (especialização, curso de extensão, pós-graduação ou residência), porém 21 $(28 \%)$ profissionais referiram não possuir esse tipo de formação, sendo 8 (38\%) da Região Metropolitana e $13(62 \%)$ das demais Regiões.
Praticamente metade da amostra de cada grupo referiu participar de algum tipo de evento na área pediátrica no último ano $(15 / 29 ; 26 / 46)$. Além disso, grande parte dos profissionais (69/75) notou evolução das práticas clínicas no tratamento fisioterapêutico nos últimos anos, mas destes, nem todos conseguiram se atualizar (6/69). Seis $(8,7 \%)$ fisioterapeutas relataram dificuldades para realizar cursos ou atualizações profissionais, sendo três de cada grupo (Região metropolitana e Demais regiões).

As maiores dificuldades mencionadas pelos profissionais para se atualizarem foram: custo elevado de cursos/formação, falta de tempo, distância e falta de incentivo da instituição empregadora. 


\section{DISCUSSÃO}

A pesar dos vários documentos do Ministério da Saúde para implementação de um sistema em redes de atenção, ainda se observa uma concentração de instituições de reabilitação em regiões economicamente mais favorecidas no estado do Rio de Janeiro. Desde $1993^{15}$, o Ministério da Saúde já documentou essa questão da concentração de serviços. A Portaria do Ministério da Saúde $n^{\circ} 793$, de 24 de abril de 2012, instituiu a Rede de Cuidados à Pessoa com Deficiência no âmbito do Sistema Único de Saúde, e destaca entre suas diretrizes, no art. $2^{\circ}$, inciso IX que haja "organização dos serviços em rede de atenção à saúde regionalizada, com estabelecimento de ações intersetoriais para garantir a integralidade do cuidado"16 com vistas a uma promoção de uma distribuição mais igualitária dos serviços entre as regiões e permitir acesso integral ao cuidado.

Mesmo com a restrição da coleta de dados aos NAIDI no município do Rio de Janeiro, a Região Metropolitana possuiu a maior concentração de instituições de tratamento. As regiões mais afastadas do centro econômico do estado, como a Noroeste Fluminense, a Médio Paraíba e a Centro-Sul Fluminense, ainda apresentavam número bem reduzido de locais de tratamento, em especial com locais próprios do SUS.

A despeito dessa concentração de assistência, considerou-se baixo o percentual de unidades municipais de saúde próprias do SUS que realizam atendimento especializado à população pediátrica. A rede de reabilitação parece pequena para garantir uma cobertura adequada aos pacientes. Com a mudança no perfil epidemiológico brasileiro na última década e aumento de doenças crônicas no Brasil, vive-se uma situação de saúde que combina uma transição demográfica acelerada e uma transição epidemiológica, em que se nota, entre outros aspectos, uma presença fortemente hegemônica das condições crônicas ${ }^{23}$ e que, portanto, necessitam de cuidados continuados, principalmente no que diz respeito à habilitação e reabilitação.

No SUS, é permitido a contratação de serviços particulares ou filantrópicos para suprir necessidades específicas de algum serviço ${ }^{17}$. Contudo, considerou-se alta a presença de instituições conveniadas que recebiam repasse do SUS $(n=16,36 \%)$. Instituições filantrópicas dependem de subsídios financeiros de pessoas física ou jurídica para o bom funcionamento, e isso pode dificultar um planejamento dos serviços em longo prazo e sua manutenção da qualidade ${ }^{17}$.

Os dados referentes aos modelos de tratamento oferecido pelas diversas instituições permitiram conhecer os serviços, nos diferentes municípios, e verificar que apesar de heterogêneos, parecem de acordo com o preconizado na literatura no que diz respeito ao tipo de tratamento oferecido, frequência de atendimento e duração das sessões ${ }^{13,18,25}$. Segundo a literatura, a metodologia de trabalho neuroevolutiva é a mais recomendada para o tratamento fisioterapêutico de crianças com sequelas neuromotoras ou atraso no desenvolvimento motor ${ }^{26-27}$. Ainda segundo as pesquisas, é recomendado que o tratamento fisioterapêutico seja realizado de forma individual, com frequência mínima de duas vezes por semana e com tempo de duração variando de 30 a 60 minutos $^{17,27}$. Os documentos oficiais existentes, chancelados pelo Ministério da Saúde brasileiro, são somente orientações para a criação de programas de tratamento ou determinações a respeito da criação e distribuição de redes de serviços. Há carência de informações oficiais que determinem diretrizes concretas sobre os programas de tratamento de estimulação precoce no que concerne à prática clínica (tipo de tratamento oferecido, frequência de atendimento e duração das sessões).

A Política Nacional de Saúde da Pessoa com Deficiência (PNSPD) preconiza um conjunto de ações destinadas a atender às necessidades dessa população no que se refere à saúde, educação, trabalho, relacionamento afetivo e social. No entanto, a implantação desta tem-se mostrado bastante incipiente no que tange a redes de atenção em reabilitação pediátrica ${ }^{11}$. Em relação à acessibilidade no SUS, apesar dos profissionais atuantes nos programas de saúde pretenderem assegurar $\mathrm{o}$ atendimento a todos os cidadãos, ainda há muito por fazer para que as pessoas com deficiência recebam a assistência equitativa, igualitária e universal preconizada ${ }^{28}$. A desarticulação intersetorial entre gestores tem dificultado a efetiva aplicação da política. Além disso, questões relacionadas com a falta de recursos humanos e de recursos orçamentários vêm sendo apontadas por gestores e profissionais como fatores limitantes.

A partir do conhecimento do perfil dos fisioterapeutas atuantes nas instituições visitadas, notou-se que que a maioria deles não possuía capacitação prévia para o exercício da atividade especializada, o que faz refletir sobre a questão da valorização do sujeito da atuação fisioterapêutica pediátrica: a criança. Por ser alguém aparentemente incapaz de estabelecer-se como sujeito de sua ação, que necessita ser tutelada por um adulto, emerge a discussão sobre a questão da autonomia da criança e do quão reconhecido esse sujeito da ação fisioterapêutica o é. Outro aspecto a ser observado é que, em geral, a fisioterapia pediátrica está associada a uma subespecialidade da fisioterapia neurofuncional ou 
pneumofuncional, e, portanto, os profissionais fisioterapeutas utilizam na pediatria modelos teóricos explicativos adaptados de outras especialidades ${ }^{29}$.

Outrossim, as dificuldades apresentadas pelos fisioterapeutas para sua capacitação profissional também são merecedoras de reflexão, a despeito de novos mecanismos de capacitação a distância e incentivo para tal.

A Organização Pan-Americana de Saúde tem colocado em pauta a discussão sobre a necessidade de melhorar a atenção prestada pelos profissionais de saúde por meio do investimento na sua capacitação ${ }^{30-31}$.

Os dados da presente pesquisa, ao apontar para essa heterogeneidade na prestação do serviço fisioterapêutico pediátrico e da capacitação dos seus profissionais, reforçam a ideia da necessidade de um programa de capacitação/educação continuada para os fisioterapeutas pediátricos.

O perfil do fisioterapeuta pode ter uma relação direta com a estruturação e organização do sistema de saúde. Segundo Tribble e colaboradores ${ }^{32}$, um importante aspecto a ser estudado com relação à formação de redes de atenção é a questão das in- tervenções de empoderamento, a partir da atuação dos profissionais de saúde envolvidos no processo e como eles realmente se sentem capacitados para seu desempenho em atendimento no contexto de problemas crônicos de saúde.

A fisioterapia em neuropediatria não é a única especialidade na qual a prestação de cuidados de saúde está ameaçada pela inadequada capacidade de equacionar a organização dos serviços e conhecimentos profissionais insuficientes. Contudo, em vista da nova demanda de crianças com CCC, a fisioterapia tem papel primordial para habilitação dessa população, possibilitando melhora da qualidade de vida e integração à sociedade.

Diante do exposto, pode-se inferir que, na luta pela garantia da integralidade na assistência fisioterapêutica no SUS, é necessário refletir, a partir da realidade percebida, sobre aspectos da organização do processo de trabalho, organização da rede de serviços e da gestão e planejamento destes, entendendo que características como acesso e qualidade dos serviços podem limitar a efetividade e resolutividade do cuidado prestado ao usuário ${ }^{33}$.

\section{CONCLUSÃO}

A fisioterapia é um dos muitos serviços que uma criança com CCC pode necessitar, e, ao longo do tempo, a importância de um melhor acesso à fisioterapia pediátrica tem sido reconhecida. Desse modo, mapear e conhecer intrinsicamente os serviços oferecidos é primordial para a gestão e para o aprimoramento do cuidado.

A despeito do avanço das condições crônicas, o sistema de saúde parece ainda não se mostrar preparado para enfrentá-las, com serviços escassos e aglomerados em regiões economicamente favorecidas. A mudança epidemiológica que vem ocorrendo requer uma nova lógica de cuidados, na direção de uma reorientação de serviços de saúde, assim como formação e capacitação de profissionais, assinalando a necessidade de investimento em uma nova pediatria ${ }^{5,34}$. Diretrizes mais concentras com relação à prática clínica fisioterapêutica ou de estimulação precoce também são necessárias para homogeneidade dos tratamentos nas diferentes instituições ${ }^{17}$.

Sabe-se, contudo, que é necessário tempo para implementar uma mudança na organização do cuidado. No entanto, existem componentes básicos importantes para que essa mudança venha a se tornar realidade, dentre os quais, destaca-se uma equipe de profissionais em número suficiente para atender à demanda, capacitada e permanentemente atualizada para o cuidado à saúde das CCC e a tantos outros desafios que possam surgir. 


\section{Referências}

1. Moreira MEL, Goldani MZ. A criança é o pai do homem: novos desafios para a área de saúde da criança. Ciên. Saúde Coletiva. 2010; 15(2):321-327.

2. Rodrigues LBB, Silva PCS, Peruhype RC, Palha PF, Popolin MP, Crispim JÁ, Pinto IC, Monroe AA, Arcêncio RA. A atenção primária à saúde na coordenação das redes de atenção: uma revisão integrativa. Ciênc. Saúde Coletiva. 2014; 19(2):343-352.

3. McPherson M, Arango P, Fox H, Lauver C, McManus M, Newacheck PW, Perrin JM, Shonkoff JP, Strickland B. A new definition of children with special health care needs. Pediatrics. 1998; 102(1 pt 1):137-140. 4. Cohen E, Kuo DZ, Agrawal R, Berry JG, Bhagat SK, Simon TD, Srivastava R. Children with medical complexity: an emerging population for clinical and research initiatives. Pediatrics. 2011; 127(3):529-533. 5. Sá MRC, Ribeiro CTM, Thomazinho PA, Cavalcanti NC, Santos FL. Reflexões sobre a rede de atenção especializada em reabilitação de crianças e adolescentes com condições crônicas de agravos à saúde. Cad Edu Saude e Fis. 2015; 2(3):67-77

6. Simeonsson RJ, Cooper DH, Sheiner AP. A Review and Analysis of the Effectiveness of Early Intervention Programs. Pediatrics. 1982; 69(5):635-640.

7. Ribeiro CTM. Perfil da fisioterapia motora em crianças portadoras da Síndrome de Down [dissertação]. Rio de Janeiro (RJ): Universidade Federal do Rio de Janeiro; 2006.

8. Doménech J, García-aymerich V, Juste J, Ortiz A. Rehabilitación motora. Revista de Neurologia 2001; 34(Supl1):S148-S150.

9. Brasil. Ministério da Saúde. Secretaria de Atenção à Saúde. Agenda de compromisso para a saúde integral e redução da mortalidade infantil, Brasília: Ministério da Saúde; 2004. 80p.

10. Brasil. Lei no 8.080 , de 19 de setembro de 1990. Dispõe sobre as condições para a promoção, proteção e recuperação da saúde, da organização e funcionamento dos serviços correspondentes e dá outras providências (Lei Orgânica da Saúde). Diário Oficial da União, Brasília, DF, 1990.

11. Brasil. Ministério da Saúde. Portaria GM/MS n. 4.279, de 30 de Dezembro de 2010, Estabelece diretrizes para a organização da Rede de Atenção à Saúde no âmbito do Sistema Único de Saúde (SUS). Brasília: Ministério da Saúde, 2010.

12. Lavras C. Atenção Primária à Saúde e a Organização de Redes Regionais de Atenção à Saúde no Brasil. Saúde Soc. 2011; 20(4):867-874.

13. Ribeiro CTM, Ribeiro MG, Araújo AP, Mello LR, Rubim LC, Ferreira JES. O sistema público de saúde e as ações de reabilitação no Brasil. Rev Panam Salud Publica. 2010; 28(1):43-48.

14. Almeida, LGR. Estudos sobre a distribuição dos serviços de reabilitação: o caso do Rio de Janeiro [dissertação]. Rio de Janeiro (RJ): Escola Nacional de Saúde Pública - Fiocruz; 2004.

15. Ministério da Saúde. Coordenação de Atenção a Grupos Especiais. Programa de Atenção à Saúde da Pessoa Portadora de Deficiência. Atenção à pessoa portadora de deficiência no Sistema Único de Saúde: planejamento e organização de serviços. Secretaria de Assistência à Saúde 1993, p. 1-48.

16. Brasil. Ministério da Saúde. Portaria $n^{\circ} 793$, de 24 de abril de 2012: Institui a Rede de Cuidados à Pessoa com Deficiência no âmbito do Sistema Único de Saúde.

17. Ribeiro CTM. Estudo da Atenção Fisioterapêutica para Crianças Portadoras de síndrome de Down no Município do Rio do Janeiro [tese]. Rio de Janeiro (RJ): Universidade Federal do Rio de Janeiro; 2010. 18. Brasil. Ministério de Educação e Cultura (MEC). Diretrizes educacionais sobre estimulação precoce: 0 portador de necessidades Especiais. Secretaria de Educação Especial. CORDE. Brasília: MEC- SEESP; 1995. 48p.

19. Brasil. Decreto № 3.298, de 20 de dezembro de 1999. Regulamenta a Lei no 7.853 , de 24 de outubro de 1989, dispõe sobre a Política Nacional para a Integração da Pessoa Portadora de Deficiência, consolida as normas de proteção, e dá outras providências. Diário Oficial da União, Brasília, DF, 1999.

20. Brasil. Ministério da saúde. Portaria № 4.279, de 30 de dezembro de 2010. Estabelece diretrizes para a organização da Rede de Atenção à Saúde no âmbito do Sistema Único de Saúde (SUS). Diário Oficial da União, Brasília, DF, 2010. 
21. Brasil. Ministério da Saúde. Portaria № 793, de 24 de abril de 2012. Institui a Rede de Cuidados à Pessoa com Deficiência no âmbito do Sistema Único de Saúde. Diário Oficial da União, Brasília, DF, 2012.

22. Cavalcanti NC. Assistência Fisioterapêutica à Osteogenesis Imperfecta no Sistema Único de Saúde do Município do Rio de Janeiro. Rio de Janeiro [dissertação]. Rio de Janeiro (RJ): Instituto Fernandes Figueira; 2013.

23. Negreiros MFV. Práticas relacionadas à detecção e intervenção nos desvios do desenvolvimento neuromotor em crianças de muito baixo peso ao nascer na cidade do Rio de Janeiro. [dissertação]. Rio de Janeiro (RJ): Instituto Fernandes Figueira; 2011.

24. Mendes EV. O cuidado das condições crônicas na atenção primária à saúde: o imperativo da consolidação da estratégia da saúde da família. Brasília: Organização Pan-Americana da Saúde, 2012. 512 p.: il. 25. Associação Brasileira de Fisioterapia Neuro Funcional - ABRAFIN. Parecer sobre estimulação precoce e Microcefalia. 2016.

26. Howle JM. Neuro-developmental Treatment Approach - Theorical Foundations and Principles of Clinical Pratice. Laguna Beach: NDTA, Inc., 381 p., 2002.

27. Uyanik M, Bumin G, Kayihan H. Comparison of different therapy approaches in children with Down syndrome. Pediatrics International 2003; 45:68-73.

28. França ISX, Pagliuca LMF. Acessibilidade das pessoas com deficiência ao sus: fragmentos históricos e desafios atuais. Rev RENE 2008; 9(2):129-137.

29. Sá MRC. A prática fisioterapêutica: reflexões sobre as ações cotidianas relacionadas à saúde da criança no ambiente hospitalar [tese]. Rio de Janeiro (RJ): Instituto Nacional de Saúde da Mulher, Criança e Adolescente Fernandes Figueira; 2013.

30. Organização Pan-Americana da Saúde (OPAS). Avaliação de tendências e prioridades sobre recursos humanos de saúde. Brasília: OPAS; 2002.

31. Ceccim RB. Educação Permanente em saúde: descentralização e disseminação de capacidade pedagógica na saúde. Cien Saude Colet 2005; 10(4):973-986.

32. Tribble DSTC, Gallagher F, Bell L, Caron C, Godbout P, Leblanc Jl; Morin P, Xhignesse M, Voyer L, Couture M. Empowerment interventions, knowledge translationand exchange: perspectives of home care professionals, clients and caregivers. BMC Health Serv Res 2008;8:177.

33. Sousa ARB, Ribeiro KSQS. A Rede Assistencial em Fisioterapia no Município de João Pessoa: uma análise a partir das demandas da atenção básica R Bras Saúde 2011; 15(3):357-368.

34. Moreira MCN, Gomes R, Sá MRC. Doenças crônicas em crianças e adolescentes: uma revisão bibliográfica. Ciênc. Saúde Coletiva 2014; 19(7):2083-2094. 